\title{
Coffee and tea consumption and risk of type 2 diabetes
}

\author{
S. van Dieren - C. S. P. M. Uiterwaal • \\ Y. T. van der Schouw • D. L. van der A • J. M. A. Boer • \\ A. Spijkerman • D. E. Grobbee • J. W. J. Beulens
}

Received: 6 May 2009 / Accepted: 7 August 2009 /Published online: 1 September 2009

(C) Springer-Verlag 2009

\begin{abstract}
Aims/hypothesis The aim of this study was to examine the association of consumption of coffee and tea, separately and in total, with risk of type 2 diabetes and which factors mediate these relations.

Methods This research was conducted as part of the Dutch Contribution to the European Prospective Investigation into Cancer and Nutrition, which involves a prospective cohort of 40,011 participants with a mean follow-up of 10 years. A validated food-frequency questionnaire was used to assess coffee and tea consumption and other lifestyle and dietary factors. The main outcome was verified incidence of type 2 diabetes. Blood pressure, caffeine, magnesium and potassium were examined as possible mediating factors.

Results During follow-up, 918 incident cases of type 2 diabetes were documented. After adjustment for potential confounders, coffee and tea consumption were both inversely associated with type 2 diabetes, with hazard ratios of 0.77 (95\% CI 0.63-0.95) for 4.1-6.0 cups of coffee per day ( $p$ for trend $=0.033)$ and $0.63(95 \%$ CI: $0.47-0.86)$ for $>5.0$ cups
\end{abstract}

S. van Dieren - C. S. P. M. Uiterwaal • Y. T. van der Schouw

D. E. Grobbee $\cdot$ J. W. J. Beulens $(\triangle)$

Julius Centre for Health Sciences and Primary Care,

University Medical Centre Utrecht,

P.O. Box 85500, 3508 GA Utrecht, the Netherlands

e-mail: J.Beulens@umcutrecht.nl

D. L. van $\operatorname{der} \mathrm{A} \cdot$ J. M. A. Boer

Centre for Nutrition and Health,

National Institute of Public Health and the Environment (RIVM),

Bilthoven, the Netherlands

A. Spijkerman · J. W. J. Beulens

Centre for Prevention and Health Services Research,

National Institute of Public Health and the Environment (RIVM),

Bilthoven, the Netherlands of tea per day ( $p$ for trend $=0.002$ ). Total daily consumption of at least three cups of coffee and/or tea reduced the risk of type 2 diabetes by approximately $42 \%$. Adjusting for blood pressure, magnesium, potassium and caffeine did not attenuate the associations.

Conclusions/interpretation Drinking coffee or tea is associated with a lowered risk of type 2 diabetes, which cannot be explained by magnesium, potassium, caffeine or blood pressure effects. Total consumption of at least three cups of coffee or tea per day may lower the risk of type 2 diabetes.

Keywords Blood pressure - Caffeine - Coffee - Magnesium • Potassium $\cdot$ Prospective study $\cdot$ Tea $\cdot$ Type 2 diabetes

$\begin{array}{ll}\text { Abbreviations } \\ \text { CVD } & \text { Cardiovascular disease } \\ \text { EPIC-NL } & \begin{array}{l}\text { Dutch Contribution to European Prospective } \\ \text { Investigation into Cancer and Nutrition }\end{array} \\ \text { FFQ } & \text { Food-frequency questionnaire } \\ \text { MORGEN } & \begin{array}{l}\text { Monitoring Project on Risk Factors for } \\ \text { Chronic Diseases }\end{array}\end{array}$

\section{Introduction}

The prevalence of type 2 diabetes mellitus has increased dramatically in the past decades and is estimated to double from 171 million people in 2000 to 366 million in 2030 [1]. The majority of diabetes cases could be prevented by changes in lifestyle and diet [2,3]. An inverse relation between coffee intake and type 2 diabetes has been reported by a number of large cohort studies [4]. The association of tea consumption with the risk of type 2 diabetes and the effects of total coffee and tea consumption are not entirely 
clear. One study did not find an association between tea intake and type 2 diabetes [5], while another study observed an association for green tea only [6].

The underlying mechanism for the relation between coffee and tea consumption and type 2 diabetes is unclear. Several components in coffee and tea have been suggested as possible causal factors. Magnesium plays a role in regulating insulin action and is inversely associated with insulin sensitivity and type 2 diabetes [7]. Potassium intake has also been associated with a reduced risk of diabetes [8]. Caffeine may affect glucose tolerance, although conflicting results have been reported. It increases energy expenditure and thermogenesis, which can stimulate insulin sensitivity [9], but short-term intervention studies reported a decreased insulin sensitivity after caffeine intake $[10,11]$. Finally, at least two studies found an inverse relation between coffee consumption and occurrence of hypertension $[12,13]$ and previous studies showed that elevated blood pressure predates type 2 diabetes and is independently associated with risk of type 2 diabetes [14, 15]. This could be explained by the inverse relation between high blood pressure and the ability of insulin to stimulate skeletal blood flow, which causes insulin resistance [16]. So far there is no conclusive evidence about whether these factors explain the inverse relation between coffee and tea intake and type 2 diabetes.

Therefore, we investigated the associations of coffee and tea consumption with risk of type 2 diabetes, and whether these relations are mediated by blood pressure and intake of potassium, magnesium and caffeine, in the Dutch Contribution to European Prospective Investigation into Cancer and Nutrition (EPIC-NL), involving a prospective cohort of 40,011 Dutch men and women. This research is an extension of an earlier investigation in which the association of coffee consumption and risk of type 2 diabetes was investigated. The current study was not only focused on coffee consumption, but the association of tea consumption and total consumption of coffee and tea with risk of type 2 diabetes was assessed. In addition, this study has six additional years of follow-up from 40,011 participants, compared with 17,000 participants, and it was based on verified cases of type 2 diabetes.

\section{Methods}

Study population and design The EPIC-NL cohort comprises the Monitoring Project on Risk Factors for Chronic Diseases (MORGEN) and Prospect cohorts [17]. The methods used are described in more detail elsewhere [18]. In brief, Prospect is a prospective population-based cohort study of 17,357 women aged 49-70 years who participated in breast cancer screening between 1993 and 1997. The
MORGEN cohort consists of men and women aged 2059 years recruited from three Dutch towns (Amsterdam, Doetinchem and Maastricht). From 1993 to 1997 each year a new random sample of about 5,000 participants was examined. These rounds of enrolment add up to 22,654 individuals. The participation rates were $34.5 \%$ for Prospect and $45 \%$ for MORGEN.

At baseline, a general questionnaire and a food-frequency questionnaire (FFQ) were mailed to all participants and these were returned at the medical examination. We excluded 246 participants with missing data on coffee and tea consumption, 615 participants with diabetes at baseline and 974 individuals without consent for linkage to disease registries, leaving 38,176 participants for this analysis.

All participants gave written informed consent prior to study inclusion. Both cohorts comply with the Declaration of Helsinki. Prospect was approved by the Institutional Review Board of the University Medical Centre Utrecht and MORGEN was approved by the Medical Ethics Committee of the Netherlands Organization for Applied Scientific Research.

General assessments The general questionnaires contained questions on demographic characteristics and risk factors for the presence of chronic diseases. Although the general questionnaires for the cohorts were not identical, similar questions were asked with slightly different answer categories. Coding of this information was standardised and merged into one uniform database. Body weight, height and waist and hip circumferences were measured. Blood pressure was measured twice on the left arm while the participants were in a supine position. The mean of the two blood pressure measurements was used in the analysis. In the Prospect study, the systolic and diastolic blood pressures were measured using a Boso Oscillomat (Bosch \& Sohn, Jungingen, Germany), while a random zero sphygmomanometer (Hawksley \& Sons, Lancing, UK) was used in the MORGEN cohort. The comparability of these different measurement procedures has been described in more detail by Schulze et al. [19]. The assessment of the Prospect cohort slightly overestimated the blood pressure compared with the MORGEN cohort.

In both cohorts daily food intake was assessed using the same validated FFQ, which included questions on the usual frequency of consumption of 77 main food groups during the year preceding enrolment. Overall, the questionnaire allows estimation of the average daily consumption of 178 foods. The FFQ was validated against 12 months of $24 \mathrm{~h}$ recalls in 121 participants before the start of the study [20, 21]. Hypertension was defined as present based on several aspects: a physician-diagnosed self-report or measured hypertension $>140 \mathrm{mmHg}$ systolic or $>90 \mathrm{mmHg}$ diastolic or the use of blood-pressure-lowering medication. Postal 
follow-up questionnaires were sent every $3-5$ years to all participants in order to detect changes in health status.

To investigate if coffee and tea consumption are also associated with biomarkers of type 2 diabetes, several biomarkers were measured in a $6.5 \%$ random sample $(n=$ $2,604)$ and in incident cases of type 2 diabetes. $\mathrm{HbA}_{1 \mathrm{c}}$ was measured in erythrocytes using an immunoturbidimetric latex test. Alanine aminotransferase, aspartate aminotransferase, gamma-glutamyltransferase, total cholesterol and triacylglycerol were measured using enzymatic methods, while high-sensitive C-reactive protein was measured with a turbidimetric method. HDL-cholesterol and LDLcholesterol were measured using homogeneous assays with enzymatic endpoints [18].

Assessment of beverages The FFQ was used to assess the amount of coffee and tea consumed daily. Participants were asked how many cups of coffee and tea on average they consumed per day/per week/per month/per year. Furthermore, participants were asked how often they consumed decaffeinated coffee with the following categories: always/ mostly (90\%); often (65\%); sometimes (35\%); and seldom/ never $(10 \%)$. These percentages were multiplied by total amount of coffee to determine how many cups of decaffeinated coffee participants drank on average. Participants were also asked how many non-alcoholic drinks they consumed and what percentage of these were caffeinated soft drinks with identical answer categories. These percentages were multiplied by total amount of non-alcoholic drinks to obtain the number of glasses of caffeinated soft drinks. Total fluid intake was calculated by adding up total intake of juices, water, milk and carbonated drinks, and excluding coffee and tea intake. We used data from the validation study [20] to estimate the reliability of the assessment of coffee and tea consumption. We observed a Spearman correlation coefficient of 0.74 for coffee consumption and 0.87 for tea consumption between the FFQ and 12 $24 \mathrm{~h}$ recalls. The coffee consumption was divided into six categories: $0-1.0$ cups (reference group); $1.1-2.0$ cups; $2.1-$ 3.0 cups; $3.1-4.0$ cups; $4.1-6.0$ cups; and $>6.0$ cups per day. Because of a different distribution of tea consumption, tea consumption was divided into five groups: $0-1.0$ cups (reference group); $1.1-2.0$ cups; $2.1-3.0$ cups; $3.1-5.0$ cups; and $>5.0$ cups per day. Finally, the number of cups of coffee and tea reported by an individual was added up and divided into five categories: $0-1.0$ cups (reference group); $1.1-3.0$ cups; 3.1-5.0 cups; 5.1-7.0 cups; and >7.0 cups of coffee and tea per day.

Assessment of type 2 diabetes The assessment of type 2 diabetes has been described in more detail elsewhere [22] The general questionnaire contained questions of whether or not a participant had been diagnosed with diabetes and, if so, additional questions were asked about the year of diagnosis and treatment. The incidence of type 2 diabetes was assessed by self-report in follow-up questionnaires. With the first follow-up questionnaire, participants received a urinary glucose strip test and were asked whether the urine strip had turned purple after $10 \mathrm{~s}$, indicating glucosuria. Also, data on the diagnosis of type 2 diabetes were obtained from the Dutch Centre for Health Care Information, which holds a standardised computerised register of hospital discharge diagnoses (all diagnoses were coded according to the International Classification of Diseases, ninth revision [ICD-9, ICD codes 250]) [23]. The records from this database were linked to the EPIC-NL cohort with a validated probabilistic method [24]. Type 2 diabetes was defined as potentially present when self-report, urinary glucose strip test or hospital discharge diagnoses reported a positive response. All potential cases of type 2 diabetes were verified against medical records of the general practitioners and pharmacists. They were asked if diabetes was diagnosed and, if so, in what year and which type. Only cases of type 2 diabetes that were verified were included as cases $(88.6 \%$ of the potential incident cases were verified). Information on vital status was obtained through linkage with the municipal administration registries.

Statistical analysis Follow-up time was calculated from the date of enrolment to the date of diabetes diagnosis or date of death; all other participants were censored at the end of follow up (January 2006). Baseline characteristics according to category of daily coffee and tea consumption were evaluated using ANOVA for continuous variables and $\chi^{2}$ test for categorical variables.

To examine the association between coffee and tea consumption and incidence of type 2 diabetes we used Cox proportional hazard regression. Hazard ratios and 95\% confidence intervals for type 2 diabetes were calculated for each category of coffee or tea intake against a reference group of low consumers (for coffee) or non-consumers (for tea), corrected for age (continuous), sex (male or female) and cohort (Prospect or MORGEN). A second multivariate model corrected for other known risk factors included smoking (present, former or never smokers), BMI $(<20.0$, $20.0-24.9,25.0-29.9$ or $\geq 30.0 \mathrm{~kg} / \mathrm{m}^{2}$ ), highest education level (low, intermediate or high), physical activity (inactive, moderately inactive, moderately active or active), family history of diabetes (present or not present), alcohol intake (continuous), daily energy intake (continuous), energyadjusted saturated fat intake (continuous), energy-adjusted intake of fibre and vitamin $\mathrm{C}$ (both continuous), hypertension and hypercholesterolaemia (present or not). A third model corrected for tea consumption (for coffee hazard ratios) and coffee consumption (for tea hazard ratios), because participants who drank a lot of coffee tended to 
drink less tea and vice versa and both beverages have been associated with the development of type 2 diabetes. Furthermore, we repeated the analysis with only four categories of coffee and tea to see if the observed associations changed significantly. Also, hazard ratios were calculated for total consumption of coffee and tea, using the multivariate adjusted model. Because results may be confounded by total fluid intake, this was checked by including it in the model with total coffee and tea consumption. Total fluid intake consisted of total intake of juices, water, milk and carbonated soft drinks. To test for a linear trend, we calculated the median for each category of coffee and tea consumption and included this as a continuous variable in the model. The square of this term was used to test for a quadratic trend.

Mediating factors were included in the multivariate model to assess the differences in hazard ratio of the linear term. The mediating factors examined were systolic and diastolic blood pressure and daily intake of magnesium, potassium and caffeine (both unadjusted and energy adjusted), all as continuous variables. To further examine the effects of caffeine, hazard ratios with $95 \%$ confidence intervals were calculated for decaffeinated coffee and caffeinated soft drinks. Interactions of coffee or tea consumption with sex, age, BMI, caffeine and smoking were explored by including the interaction term of any of these combinations in the multivariate model using a likelihood ratio test. Furthermore, the association between several biomarkers for type 2 diabetes and coffee and tea consumption has been examined by conducting a linear regression analysis corrected for the same variables as used in the multivariate Cox model.

The Cox proportional hazards assumption was examined by visually inspecting $\log -\log$ plots with no deviations detected. $p$ values $<0.05$ from two-tailed analyses were considered statistically significant. All statistical analyses were performed using SPSS for Windows version 14.0 (SPSS, Chicago, IL, USA).

\section{Results}

As coffee consumption increased, so did BMI, alcohol consumption, energy intake and prevalence of smoking, but tea consumption decreased (Table 1). Increasing tea consumption was associated with a reduction in alcohol consumption, BMI, energy intake and physical activity (Table 2).

During a mean follow-up of 10 years, 918 incident cases of type 2 diabetes were documented. A U-shaped association between coffee consumption and risk of type 2 diabetes was observed (Table 3). Adjusting for tea consumption lowered the hazard ratios, with the lowest hazard ratio for $4.1-6.0$ cups of coffee/day $(0.74$ [95\% CI

Table 1 Baseline characteristics by daily coffee and tea consumption in 38,176 participants

\begin{tabular}{|c|c|c|c|c|c|c|}
\hline \multirow[t]{2}{*}{ Characteristic } & \multicolumn{6}{|c|}{ Coffee consumption (cups per day) } \\
\hline & $0-1.0$ & $1.1-2.0$ & $2.1-3.0$ & $3.1-4.0$ & $4.1-6.0$ & $>6.0$ \\
\hline Participants $(n)$ & 7,919 & 4,763 & 5,148 & 6,597 & 9,034 & 4,715 \\
\hline Male participants, $\%(n)$ & $21.4 \%(1,697)$ & $19.1 \%(910)$ & $20.8 \%(1,072)$ & $20.0 \%(1,319)$ & $29.9 \%(2,702)$ & $44.6 \%(2,105)$ \\
\hline Age, years (SD) & $46.5(14.0)$ & $49.6(12.5)$ & $49.9(11.9)$ & $51.8(11.0)$ & $49.7(10.5)$ & $47.1(9.9)$ \\
\hline Caffeine intake, mg/day (SD) & $203.7(126.0)$ & $287.7(102.9)$ & $349.3(89.4)$ & $431.7(85.6)$ & $523.0(84.1)$ & $754.3(184.8)$ \\
\hline Tea intake, cups/day (SD) & $3.6(3.0)$ & $2.9(2.4)$ & $2.4(2.0)$ & $2.4(2.0)$ & $1.7(1.8)$ & $1.0(1.6)$ \\
\hline Systolic blood pressure, $\mathrm{mmHg}(\mathrm{SD})$ & $124.0(19.3)$ & $126.0(19.8)$ & $126.8(19.1)$ & $128.3(19.2)$ & $126.6(18.1)$ & $124.3(16.5)$ \\
\hline Diastolic blood pressure, $\mathrm{mmHg}(\mathrm{SD})$ & $76.7(10.9)$ & $77.3(10.9)$ & $77.9(10.5)$ & $78.5(10.6)$ & $78.3(10.4)$ & $78.0(10.2)$ \\
\hline Ethanol intake, g/week (SD) & $54.0(90.0)$ & $74.0(113.0)$ & $77.8(99.5)$ & $75.7(104.1)$ & $88.9(112.8)$ & $100.8(131.4)$ \\
\hline Current smoker, \% $(n)$ & $22.5(1,784)$ & $22.6(1,078)$ & $25.3(1,303)$ & $25.0(1,649)$ & $36.6(3,310)$ & $53.9(2,543)$ \\
\hline Low education level, \% $(n)$ & $53.8(4,260)$ & $51.9(2,473)$ & $52.3(2,693)$ & $62.3(4,109)$ & $60.7(5,487)$ & $59.5(2,807)$ \\
\hline BMI, kg/m² (SD) & $25.5(4.2)$ & $25.2(3.9)$ & $25.4(3.9)$ & $25.7(4.0)$ & $25.9(3.9)$ & $26.0(4.0)$ \\
\hline Low physical activity, \% (n) & $10.5(828)$ & $8.9(423)$ & $8.6 \%(441)$ & $7.3 \%(484)$ & $8.6(781)$ & $11.9(562)$ \\
\hline Total energy intake, kJ/day (SD) & $8,261(2,730)$ & $8,335(2,458)$ & $8,416(2,435)$ & $8,428(2,415)$ & $8,833(2,656)$ & $9,645(3,219)$ \\
\hline Saturated fat intake $^{\mathrm{a}}, \mathrm{g} /$ day (SD) & $31.4(6.0)$ & $32.1(5.8)$ & $32.4(5.6)$ & $33.0(5.7)$ & $33.1(5.7)$ & $33.5(6.2)$ \\
\hline Magnesium intake $^{\mathrm{a}}, \mathrm{mg} /$ day (SD) & $322.8(53.3)$ & $328.1(47.6)$ & $332.8(45.8)$ & $336.3(44.1)$ & $342.3(45.4)$ & $353.3(49.2)$ \\
\hline Potassium intake $^{\mathrm{a}}, \mathrm{mg} /$ day (SD) & $3,419(622)$ & $3,486(546)$ & $3,551(524)$ & $3,618(508)$ & $3,698(517)$ & $3,857(554)$ \\
\hline
\end{tabular}

${ }^{\mathrm{a}}$ Energy-adjusted

${ }^{*}$ All $p$ values are $<0.05$ for the comparison between coffee consumption categories 
Table 2 Baseline characteristics by daily tea consumption in 38,176 participants

\begin{tabular}{|c|c|c|c|c|c|}
\hline \multirow[t]{2}{*}{ Characteristic } & \multicolumn{5}{|c|}{ Tea consumption (cups per day) } \\
\hline & $0-1.0$ & $1.1-2.0$ & $2.1-3.0$ & $3.1-5.0$ & $>5.0$ \\
\hline Participants (n) & 15,873 & 7,670 & 4,211 & 6,469 & 3,953 \\
\hline Male participants, \% (n) & $36.4(5,777)$ & $23.1(1,774)$ & $18.1(762)$ & $14.7(949)$ & $13.7(543)$ \\
\hline Age, years (SD) & $46.9(11.7)$ & $49.8(11.6)$ & $50.4(12.0)$ & $52.0(11.7)$ & $50.4(12.1)$ \\
\hline Caffeine intake, mg/day (SD) & $411.7(231.7)$ & $392.6(187.3)$ & $390.8(177.7)$ & $427.3(164.7)$ & $494.6(182.5)$ \\
\hline Tea intake, cups/day (SD) & $4.3(2.7)$ & $3.4(2.1)$ & $2.9(2.0)$ & $2.6(1.9)$ & $1.9(1.9)$ \\
\hline Systolic blood pressure, $\mathrm{mmHg}$ (SD) & $125.3(17.8)$ & $126.3(19.2)$ & $126.4(19.2)$ & $127.6(19.5)$ & $125.5(19.8)$ \\
\hline Diastolic blood pressure, $\mathrm{mmHg}(\mathrm{SD})$ & $78.2(10.5)$ & $77.7(10.7)$ & $77.5(10.8)$ & $77.7(10.6)$ & $76.8(10.8)$ \\
\hline Ethanol intake, $\mathrm{g} /$ week (SD) & $90.1(121.6)$ & $73.8(101.1)$ & $69.0(91.7)$ & $65.0(94.1)$ & $63.7(101.8)$ \\
\hline Current smoker, \% (n) & $41.4(6,579)$ & $28.0(2,147)$ & $22.2(933)$ & $18.9(1,223)$ & $19.9(785)$ \\
\hline Low education level, \% (n) & $59.9(9,509)$ & $59.8(4,588)$ & $55.9(2,354)$ & $54.4(3,522)$ & $47.2(1,864)$ \\
\hline BMI, kg/m² (SD) & $25.9(4.1)$ & $25.7(4.0)$ & $25.4(3.9)$ & $25.4(3.8)$ & $25.0(3.9)$ \\
\hline Low physical activity, \% (n) & $11.3(1,800)$ & $8.4(648)$ & $7.6(318)$ & $6.7(433)$ & $8.1 \%(321)$ \\
\hline Total energy intake, kJ/day (SD) & $8,956(2,898)$ & $8,535(2,593)$ & $8,366(2,456)$ & $8,324(2,393)$ & $8,253(2,569)$ \\
\hline Saturated fat intake ${ }^{\mathrm{a}}, \mathrm{g} /$ day (SD) & $32.8(6.0)$ & $32.7(5.8)$ & $32.5(5.7)$ & $32.4(5.7)$ & $31.9(6.0)$ \\
\hline Magnesium intake $^{\mathrm{a}}, \mathrm{mg} /$ day (SD) & $333.3(50.2)$ & $335.3(46.9)$ & $335.7(46.1)$ & $338.6(46.6)$ & $339.6(50.8)$ \\
\hline Potassium intake $^{\mathrm{a}}, \mathrm{mg} /$ day (SD) & $3,620(572)$ & $3,603(548)$ & $3,578(548)$ & $3,594(553)$ & $3,545(603)$ \\
\hline
\end{tabular}

${ }^{\text {a }}$ Energy-adjusted

${ }^{*}$ All $p$ values are $<0.05$ for the comparison between tea consumption categories

$\left.0.61-0.91], p_{\text {trend }}=0.019\right)$. A strong inverse association was observed between tea consumption and risk of type 2 diabetes (Table 4). After multivariate adjustments and adjusting for coffee consumption the lowest hazard ratio was $0.68(95 \%$ CI $0.52-0.89)$ for $>5.0$ cups of tea/day $\left(p_{\text {trend }}=0.01\right)$. Substituting waist circumference for BMI did not alter the results (HR coffee 0.74 [95\% CI 0.61-0.91]).

There were 8,819 people who drank $>1.0$ cup of decaffeinated coffee/day and 228 participants in this group developed type 2 diabetes. We found no association between decaffeinated coffee intake and risk of type 2 diabetes (1.13 [95\% CI 0.76-1.67] for $>1.0$ cup of decaffeinated coffee/day) or between caffeinated soft drinks and risk of type 2 diabetes (1.64 [0.60-4.49] for drinking $>3.0$ glasses of caffeinated soft drink/day). Excluding hypertensive patients resulted in similar hazard ratios for tea consumption and even stronger associations for coffee consumption (HR coffee 0.62 [95\% CI 0.46$0.83]$, HR tea 0.70 [95\% CI 0.48-1.02]). Stronger associations were also observed when patients with hyperlipidaemia, hypertension and prevalent cases of CVD were excluded (HR coffee 0.55 [95\% CI $0.40-0.77]$, HR tea 0.60 [95\% CI $0.39-0.92])$. Similar associations occurred when coffee and tea consumption were both divided into four categories (HR 0.75 [95\% CI 0.61-0.92] for $>5.0$ cups of coffee).

Table 3 Daily coffee consumption and hazard ratios $(95 \%$ CI) for the risk of type 2 diabetes among 38,176 Dutch men and women

\begin{tabular}{|c|c|c|c|c|c|c|c|c|}
\hline \multirow[t]{2}{*}{ Hazard ratio } & \multicolumn{6}{|c|}{ Coffee consumption (cups per day) } & \multirow{2}{*}{$\begin{array}{l}p \text { value } \\
\text { linear } \\
\text { trend }\end{array}$} & \multirow{2}{*}{$\begin{array}{l}p \text { value } \\
\text { quadratic } \\
\text { trend }\end{array}$} \\
\hline & $0-1.0$ & $1.1-2.0$ & $2.1-3.0$ & $3.1-4.0$ & $4.1-6.0$ & $>6.0$ & & \\
\hline Cases, $\mathrm{n}$ & 206 & 109 & 134 & 152 & 204 & 113 & - & - \\
\hline $\begin{array}{l}\text { HR adjusted for } \\
\text { sex, age, BMI and } \\
\text { cohort }(95 \% \text { CI) }\end{array}$ & 1.00 & $0.85(0.67-1.07)$ & $0.96(0.77-1.19)$ & $0.73(0.59-0.90)$ & $0.78(0.64-0.94)$ & $0.91(0.72-1.15)$ & 0.089 & 0.016 \\
\hline $\begin{array}{l}\text { Multivariate-adjusted } \\
\text { HR }(95 \% \mathrm{CI})^{\mathrm{a}}\end{array}$ & 1.00 & $0.89(0.70-1.12)$ & $0.98(0.79-1.22)$ & $0.77(0.62-0.95)$ & $0.80(0.65-0.97)$ & $0.93(0.73-1.19)$ & 0.13 & 0.040 \\
\hline $\begin{array}{l}\text { HR further adjusted } \\
\text { for tea }(95 \% \mathrm{CI})^{\mathrm{a}}\end{array}$ & 1.00 & $0.88(0.69-1.11)$ & $0.94(0.75-1.17)$ & $0.75(0.60-0.92)$ & $0.74(0.61-0.910)$ & $0.84(0.65-1.08)$ & 0.019 & 0.045 \\
\hline
\end{tabular}

${ }^{a}$ Adjusted for cohort, sex, age, BMI, highest education, physical activity, family history of diabetes, smoking, alcohol intake, energy intake, energy-adjusted saturated fat intake, energy-adjusted fibre intake, energy-adjusted vitamin $\mathrm{C}$ intake, hypercholesterolaemia and hypertension 
Table 4 Daily tea consumption and hazard ratios $(95 \% \mathrm{CI})$ for the risk of type 2 diabetes among 38,176 Dutch men and women

\begin{tabular}{|c|c|c|c|c|c|c|c|}
\hline \multirow[t]{2}{*}{ Hazard ratio } & \multicolumn{5}{|c|}{ Tea consumption (cups per day) } & \multirow{2}{*}{$\begin{array}{l}p \text { value } \\
\text { linear trend }\end{array}$} & \multirow{2}{*}{$\begin{array}{l}p \text { value } \\
\text { quadratic trenc }\end{array}$} \\
\hline & $0-1.0$ & $1.1-2.0$ & $2.1-3.0$ & $3.1-5.0$ & $>5.0$ & & \\
\hline Cases, $\mathrm{n}$ & 414 & 170 & 110 & 152 & 72 & - & - \\
\hline $\begin{array}{l}\text { HR adjusted for } \\
\text { sex, age, } \mathrm{BMI} \text { and } \\
\text { cohort }(95 \% \mathrm{CI})\end{array}$ & 1.00 & $0.77(0.64-0.92)$ & $0.93(0.75-1.15)$ & $0.78(0.65-0.95)$ & $0.71(0.55-0.91)$ & 0.018 & 0.139 \\
\hline $\begin{array}{l}\text { Multivariate-adjusted } \\
\text { HR }(95 \% \mathrm{CI})^{\mathrm{a}}\end{array}$ & 1.00 & $0.77(0.64-0.93)$ & $0.93(0.75-1.15)$ & $0.79(0.65-0.96)$ & $0.73(0.57-0.95)$ & 0.041 & 0.134 \\
\hline $\begin{array}{l}\text { HR further adjusted } \\
\text { for coffee }(95 \% \mathrm{CI})^{\mathrm{a}}\end{array}$ & 1.00 & $0.77(0.64-0.92)$ & $0.89(0.72-1.11)$ & $0.77(0.63-0.94)$ & $0.68(0.52-0.89)$ & 0.014 & 0.116 \\
\hline
\end{tabular}

${ }^{a}$ Adjusted for cohort, sex, age, BMI, highest education, physical activity, family history of diabetes, smoking, alcohol intake, energy intake, energy-adjusted saturated fat

Using the data on total amount of coffee and tea consumed resulted in a stronger inverse association with the risk of type 2 diabetes (Fig. 1). Consumption of at least three cups of coffee and/or tea per day was associated with a reduced risk of type 2 diabetes of approximately $42 \%$ (HR 0.58 [95\% CI $0.42-$ $\left.0.80], p_{\text {trend }}<0.001\right)$. Drinking more than three cups of coffee and/or tea did not substantially lower the hazard ratio any further. The relative amount of coffee vs tea did not affect the associations for total consumption.

Similar associations were observed when we adjusted for total fluid consumption, the hazard ratio for type 2 diabetes was 0.53 (95\% CI $0.39-0.73)$ for consumption of $>7.0$ cups of coffee and tea/day $\left(p_{\text {trend }}<0.001\right)$. Excluding cases obtained in the first 4 years, to prevent reverse causation, did not significantly alter the observed associations for tea

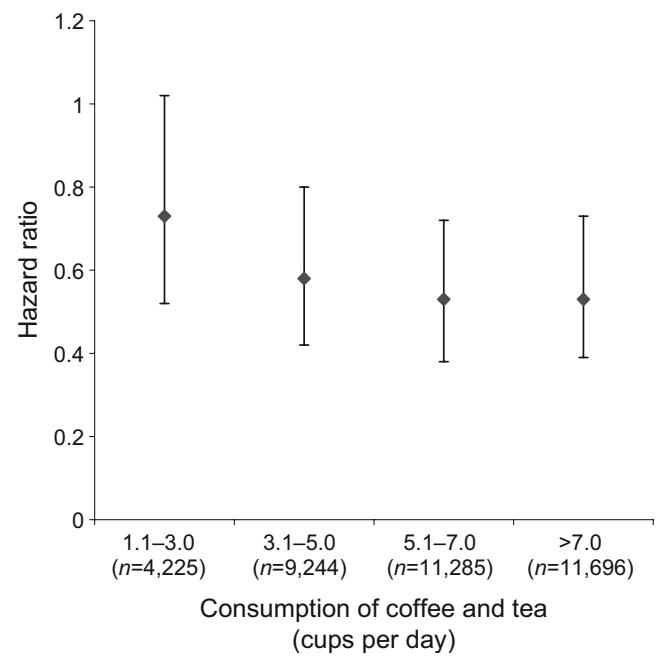

Fig. 1 Hazard ratios $(95 \% \mathrm{CI})$ for the risk of type 2 diabetes by daily total consumption of coffee and tea among 38,176 Dutch men and women. Adjusted for cohort, sex, age, BMI, highest education, physical activity, family history of diabetes, smoking, alcohol intake, energy intake, energy-adjusted saturated fat intake, energy-adjusted fibre intake, energy-adjusted vitamin $\mathrm{C}$ intake, hypercholesterolaemia and hypertension
(HR 0.64 [95\% CI 0.46-0.89]) and lowered the hazard ratio for coffee further (HR 0.72 [95\% CI 0.57-0.90]).

Adjusting for several mediating factors did not alter the results. The hazard ratios for 4.1-6.0 cups of coffee were similar after adjusting for diastolic and systolic blood pressure $(0.76$ [95\% CI $0.62-0.94])$, for magnesium and potassium $(0.75$ [ $95 \%$ CI $0.62-0.93])$ and for caffeine $(0.73$ [95\% CI $0.50-1.07])$. The hazard ratio for $>6.0$ cups of tea was 0.64 (95\% CI $0.47-0.86)$ after adjusting for diastolic blood pressure and potassium, 0.63 (95\% CI $0.47-0.86$ ) after adjusting for systolic blood pressure and magnesium, and for caffeine 0.61 (95\% CI $0.42-0.90)$. No significant interactions of coffee or tea consumption with sex, age, BMI and smoking were observed. We observed no significant association between coffee or tea consumption and any of the examined biomarkers.

\section{Discussion}

In this cohort of 38,176 men and women, both coffee and tea consumption were associated with a lowered risk of type 2 diabetes. Consumption of at least three cups of coffee and/or tea was associated with a lowered risk of type 2 diabetes. Blood pressure and intake of magnesium, potassium and caffeine did not explain these associations.

The strengths of this study are its large sample size, prospective design, validation of diabetes cases and extensive information about lifestyle and diet of the participants, but some limitations need to be addressed. First, we relied on self-reported coffee and tea consumption and therefore misclassification may have occurred. However, we validated the assessment of coffee and tea consumption, showing correlations over 0.75 . A second limitation of our study is the assessment of decaffeinated coffee. Participants could only indicate in categories how much decaffeinated coffee they consumed. Therefore, we could not accurately assess the effect of decaffeinated 
coffee. Similarly, no specific information on green tea consumption was available. However, only $4.6 \%$ of the total amount of tea consumed in the Netherlands is green tea [25].

Second, the presence of type 2 diabetes is often undetected, and may remain preclinical for up to 9-12 years [26]. Patients with undetected type 2 diabetes in our cohort may have been misclassified as people without diabetes, resulting in attenuation of associations. As a sensitivity analysis we excluded prevalent cases of cardiovascular disease, hypertension or hyperlipidaemia, which resulted in slightly stronger estimates.

Third, participants may have changed their coffee and tea consumption over the years because of their health. Excluding cases obtained in the first 4 years did not alter the observed associations.

Last, coffee and tea drinkers have very different — almost opposite - health behaviour. Coffee drinkers tend to smoke more and have a less healthy diet, while tea consumption is associated with a healthier lifestyle. Such confounding could attenuate the observed relation, particularly for tea consumption, while it could partly explain the increased risks in the higher-intake categories for coffee consumption. Although we have adjusted for several lifestyle and dietary factors, we cannot exclude residual confounding.

The observed reduction in risk of type 2 diabetes with higher coffee consumption is in line with previous studies [4, 27-30], although our study did not indicate a strong dose-response relation. In our study population, high coffee consumption was strongly related to low tea consumption. Associations unadjusted for tea consumption could therefore be confounded by lower consumption of tea in the high-intake groups, leading to higher risks. These results could then reflect the effect of lower tea consumption instead of higher coffee consumption. Most studies on coffee consumption did not account for tea consumption [28-30]. However, this cannot solely explain the increased risks in the higher categories of coffee consumption. Another explanation could be residual confounding by unhealthy lifestyles in the categories of higher coffee consumption. In addition, it could be explained by higher intake of ingredients associated with increased risks of type 2 diabetes, such as kahweol and cafestol [31].

Several studies have investigated the associations between tea consumption and type 2 diabetes. Some of these studies did not find any associations [27, 29, 30, 32], while one study observed an inverse relation for both coffee and black tea consumption and type 2 diabetes but not for green tea [32]. Another study found an association only for green tea, not for black or oolong tea [6]. A recent meta-analysis summarised these studies and concluded that consumption of at least four cups of tea may lower the risk of type 2 diabetes, but a reduced risk was not observed for one to three cups of tea per day [33]. In addition, they were unable to distinguish between black and green tea in this meta-analysis.
We observed an inverse association between tea consumption and type 2 diabetes over the entire range, even though the hazard ratio for two to three cups/day was slightly higher. Although we could not differentiate between black and green tea, an estimated $95 \%$ of the total tea consumption is black tea in the Netherlands [25]. Recently, a study reported that both black and green tea lower blood glucose concentrations [34]. Combined with our results, it seems likely that the association between tea consumption and type 2 diabetes is not solely due to consumption of green tea.

Moreover, consumption of at least three cups of coffee and/or tea was associated with a lowered risk of type 2 diabetes. A similar inverse relation for combined consumption of coffee and tea has been observed in the Whitehall II study [5]. Unfortunately, these analyses were limited by small sample size $(n=2,300)$, and coffee and tea consumption was divided into only two categories, therefore no effect of consuming a greater amount of coffee and tea could be observed [5].

Blood pressure and intake of magnesium, potassium and caffeine were included in our model to assess whether these factors mediated the observed association, but none affected the associations substantially. One study observed a borderline inverse association between magnesium intake and risk of type 2 diabetes. However, magnesium did not explain the relation between coffee and type 2 diabetes in our study, similar to other studies [30, 34-36]. In our study up to three cups of coffee was associated with higher blood pressure, but more than four cups of coffee was associated with a lowered blood pressure. However, the inclusion of blood pressure in the model did not explain the association between coffee or tea consumption and type 2 diabetes. Caffeine intake only slightly attenuated the relation between coffee consumption and type 2 diabetes, but the correlation between coffee and caffeine was 0.86 , making it difficult to distinguish the effects of coffee and caffeine. Furthermore, no association between several biomarkers and coffee and tea consumption has been observed.

These results suggest that other factors may be more important to explain the relation of coffee and tea consumption with type 2 diabetes. The effect of total coffee and tea consumption could indicate that the beneficial effect of coffee and tea consumption is due to their antioxidant content. Coffee is rich in the antioxidant chlorogenic acid and tea contains flavonols and flavones as antioxidants [37, 38]. Antioxidants may reduce the amount of reactive oxygen species, which activate stress-sensitive pathways. These pathways can lead to insulin resistance, impaired insulin secretion and beta cell dysfunction [39, 40].

This relation is still controversial, as studies examining dietary and serum antioxidants with type 2 diabetes show inconsistent results ranging from no relation to an inverse 
relation for certain antioxidants [41, 42]. A recent randomised crossover trial, however, showed that chlorogenic acid and trigonelline significantly lower glucose and insulin [37]. This trial supports a mechanism involving these components, but more trials are needed to further elucidate these effects.

In conclusion, the findings in this large prospective cohort study show an inverse association of both coffee and tea consumption with risk of type 2 diabetes. Blood pressure and intake of magnesium, caffeine and potassium did not explain these associations. A stronger inverse association was observed when total coffee and tea consumption was investigated. Daily consumption of at least three cups of coffee and/or tea may be associated with a lowered risk of type 2 diabetes.

Acknowledgements This study was funded by an unrestricted grant from the Institute for Scientific Information on Coffee/Physiological Effects of Coffee Committee. The sponsor of the study had no role in study design, data collection, data analysis, data interpretation or writing of the report.

Duality of interests The authors declare that there is no duality of interest associated with this manuscript.

\section{References}

1. Wild S, Roglic G, Green A, Sicree R, King H (2004) Global Prevalence of Diabetes: estimates for the year 2000 and projections for 2030. Diabetes Care 27:1047-1053

2. Hu FB, Manson JE, Stampfer MJ et al (2001) Diet, lifestyle, and the risk of type 2 diabetes mellitus in women. $\mathrm{N}$ Engl J Med 345:790-797

3. Lindström J, Ilanne-Parikka P, Peltonen M et al (2006) Sustained reduction in the incidence of type 2 diabetes by lifestyle intervention: follow-up of the Finnish Diabetes Prevention Study. Lancet 368:1673-1679

4. Van Dam RM, Hu FB (2005) Coffee consumption and risk of type 2 diabetes: a systematic review. J Am Med Assoc 294:97-104

5. Hamer M, Witte DR, Mosdol A, Marmot MG, Brunner EJ (2008) Prospective study of coffee and tea consumption in relation to risk of type 2 diabetes mellitus among men and women: The Whitehall II study. Br J Nutr 1046-1053

6. Iso H, Date C, Wakai K et al (2006) The relationship between green tea and total caffeine intake and risk for self-reported type 2 diabetes among Japanese adults. Ann Intern Med 144:554-562

7. Barbagallo M, Dominguez LJ (2007) Magnesium metabolism in type 2 diabetes mellitus, metabolic syndrome and insulin resistance. Arch Biochem Biophys 458:40-47

8. Colditz GA, Manson JE, Stampfer MJ, Rosner B, Willett WC, Speizer FE (1992) Diet and risk of clinical diabetes in women. Am J Clin Nutr 55:1018-1023

9. Park S, Jang JS, Hong SM (2007) Long-term consumption of caffeine improves glucose homeostasis by enhancing insulinotropic action through islet insulin/insulin-like growth factor 1 signaling in diabetic rats. Metabolism 56:599-607

10. Keijzers GB, de Galan BE, Tack CJ, Smits P (2002) Caffeine can decrease insulin sensitivity in humans. Diabetes Care 25:364-369

11. Lane JD, Barkauskas CE, Surwit RS, Feinglos MN (2004) Caffeine impairs glucose metabolism in type 2 diabetes. Diabetes Care 27:2047-2048
12. Uiterwaal CSPM, Verschuren WMM, Bueno-de-Mesquita HB et al (2007) Coffee intake and incidence of hypertension. Am J Clin Nutr 85:718-723

13. Winkelmayer WC, Stampfer MJ, Willett WC, Curhan GC (2005) Habitual caffeine intake and the risk of hypertension in women. $\mathrm{J}$ Am Med Assoc 294:2330-2335

14. Stolk RP, van Splunder IP, Schouten JS, Witteman JC, Hofman A, Grobbee DE (1993) High blood pressure and the incidence of non-insulin dependent diabetes mellitus: findings in a 11.5 year follow-up study in The Netherlands. Eur J Epidemiol 9:134-139

15. Meisinger C, Doring A, Heier M (2008) Blood pressure and risk of type 2 diabetes mellitus in men and women from the general population: the Monitoring Trends and Determinants on Cardiovascular Diseases/Cooperative Health Research in the Region of Augsburg Cohort Study. J Hypertens 26:1809-1815

16. Baron AD, Brechtel-Hook G, Johnson A, Hardin D (1993) Skeletal muscle blood flow. A possible link between insulin resistance and blood pressure. Hypertension 21:129-135

17. Riboli E, Kaaks R (1997) The EPIC Project: rationale and study design. Int J Epidemiol 26:S6-S14

18. Beulens JW, Monninkhof EM, Verschuren MW et al (2009) Cohort profile: The EPIC-NL study. Int J Epidemiol doi:10.1093/ ije/dyp 217

19. Schulze MB, Kroke A, Saracci R, Boeing H (2002) The effect of differences in measurement procedure on the comparability of blood pressure estimates in multi-centre studies. Blood Press Monit 7:95-104

20. Ocké MC, Bueno-de-Mesquita HB, Goddijn HE et al (1997) The Dutch EPIC Food Frequency Questionnaire. I. Description of the questionnaire, and relative validity and reproducibility for food groups. Int J Epidemiol 26:S37-S48

21. Ocké MC, Bueno-de-Mesquita HB, Pols MA, Smit HA, van Staveren WA, Kromhout D (1997) The Dutch EPIC Food Frequency Questionnaire. II. Relative validity and reproducibility for nutrients. Int J Epidemiol 26:S49-S58

22. Beulens JWJ, Stolk RP, van Der Schouw YT, Grobbee DE, Hendriks HFJ, Bots ML (2005) Alcohol consumption and risk of type 2 diabetes among older women. Diabetes Care 28:2933-2938

23. ICDData.com. ICD-9-CM Diagnosis 250. Available from www. icd9data.com/2007/Volume1/240-279/250-259/250/default.htm, accessed 18 August 2009

24. Herings RMC, Bakker A, Stricker BHC, Nap G (1992) Pharmacomorbidity linkage: a feasibility study comparing morbidity in two pharmacy based exposure cohorts. J Epidemiol Community Health 46:136-140

25. Vereniging van nederlandse koffiebranders en theepakkers (2006) Jaarverslag 2006. Available from www.vnkt.nl/vnkt/pdf/VNKT\% 20Jaarverslag\%202006.pdf, accessed 18 August 2009

26. Harris MI, Klein R, Welborn TA, Knuiman MW (1992) Onset of NIDDM occurs at least 4-7 yr before clinical diagnosis. Diabetes Care 15:815-819

27. Salazar-Martinez E, Willett WC, Ascherio A et al (2004) Coffee consumption and risk for type 2 diabetes mellitus. Ann Intern Med $140: 1-8$

28. Tuomilehto J, Hu G, Bidel S, Lindstrom J, Jousilahti P (2004) Coffee consumption and risk of type 2 diabetes mellitus among middleaged Finnish men and women. J Am Med Assoc 291:1213-1219

29. Van Dam RM, Willett WC, Manson JE, Hu FB (2006) Coffee, caffeine, and risk of type 2 diabetes: A prospective cohort study in younger and middle-aged U.S. women. Diabetes Care 29:398-403

30. Pereira MA, Parker ED, Folsom AR (2006) Coffee consumption and risk of type 2 diabetes mellitus: An 11-year prospective study of 28812 postmenopausal women. Arch Int Med 166:1311-1316

31. Ranheim T, Halvorsen B (2005) Coffee consumption and human health-beneficial or detrimental?-Mechanisms for effects of coffee consumption on different risk factors for cardiovascular disease and type 2 diabetes mellitus. Mol Nutr Food Res 49:274-284 
32. Odegaard AO, Pereira MA, Koh WP, Arakawa K, Lee HP, Yu MC (2008) Coffee, tea, and incident type 2 diabetes: the Singapore Chinese Health Study. Am J Clin Nutr 88:979-985

33. Jing Y, Han G, Hu Y, Bi Y, Li L, Zhu D (2009) Tea consumption and risk of type 2 diabetes: a meta-analysis of cohort studies. J Gen Intern Med 24:557-562

34. Polychronopoulos E, Zeimbekis A, Kastorini CM et al (2008) Effects of black and green tea consumption on blood glucose levels in non-obese elderly men and women from Mediterranean Islands (MEDIS epidemiological study). Eur J Nutr 47:10-16

35. Greenberg JA, Axen KV, Schnoll R, Boozer CN (2005) Coffee, tea and diabetes: the role of weight loss and caffeine. Int $\mathrm{J}$ Obes (Lond) 29:1121-1129

36. Van Dam RM, Hu FB, Rosenberg L, Krishnan S, Palmer JR (2006) Dietary calcium and magnesium, major food sources, and risk of type 2 diabetes in U.S. black women. Diabetes Care 29:2238-2243

37. van Dijk AE, Olthof MR, Meeuse JC, Seebus E, Heine RJ, van Dam RM (2009) Acute effects of decaffeinated coffee and the major coffee components chlorogenic acid and trigonelline on glucose tolerance. Diabetes Care 32:1023-1025

38. Song Y, Manson JE, Buring JE, Sesso HD, Liu S (2005) Associations of dietary flavonoids with risk of type 2 diabetes, and markers of insulin resistance and systemic inflammation in women: a prospective study and cross-sectional analysis. J Am Coll Nutr 24:376-384

39. Evans JL, Goldfine ID, Maddux BA, Grodsky GM (2003) Are oxidative stress-activated signaling pathways mediators of insulin resistance and beta-cell dysfunction? Diabetes 52:1-8

40. Tunnicliffe JM, Shearer J (2008) Coffee, glucose homeostasis, and insulin resistance: physiological mechanisms and mediators. Appl Physiol Nutr Metab 33:1290-1300

41. Montonen J, Knekt P, Rvinen R, Reunanen A (2004) Dietary antioxidant intake and risk of type 2 diabetes. Diabetes Care 27:362-366

42. Liu S, Ajani U, Chae C, Hennekens C, Buring JE, Manson JE (1999) Long-term beta-carotene supplementation and risk of type 2 diabetes mellitus: a randomized controlled trial. JAMA 282:1073-1075 